\title{
MOLECULAR HYDROGEN EMISSION IN NGC 7027
}

\author{
S. BeCKWITH, ${ }^{\text {a) }}$ G. NeUgebauer, E. E. BeCKLiN, ${ }^{\text {b) }}$ and K. MATthews \\ Palomar Observatory, California Institute of Technology, Pasadena, California 91125
}

\section{S. E. PERSSON}

Mount Wilson and Las Campanas Observatories, Carnegie Institution of Washington, 813 Santa Barbara Street, Pasadena, California 91101 Received 25 January 1980; revised 3 April 1980

\begin{abstract}
The spatial distribution of the emission in the $v=1 \rightarrow 0 S(1)$ line of $\mathrm{H}_{2}$ in the planetary nebula NGC 7027 is presented. The excited $\mathrm{H}_{2}$ molecules do not fill the same volume as the ionized gas and most likely reside near the outer edge of the nebula. The spatial resolution of the data, $5^{\prime \prime}$, is insufficient to define the location of the molecules precisely. An upper limit to the strength of the $v=2 \rightarrow 1 S(1)$ line is given; it is low enough to preclude simple ultraviolet fluorescence as the source of excitation. A simple shock model can fit the line ratio data, however, and there is enough energy in the expanding nebula to sustain such a shock. A rough estimate of $1 M_{\odot}$ to $4 M_{\odot}$ for the mass in the molecular cloud surrounding NGC 7027 is derived.
\end{abstract}

\section{INTRODUCTION}

Emission from molecular hydrogen in a planetary nebula was first detected from NGC 7027 by Treffers et al. (1976). This planetary nebula, like NGC 6720 (Beckwith, Persson, and Gatley 1978) is sufficiently extended that measurements of the spatial distribution of the emission of $\mathrm{H}_{2}$ can be made. In this paper we present spatial data on the emission in the $\mathrm{H}_{2} v=1 \rightarrow$ $0 S(1)$ and $\mathrm{H}$ I $\mathrm{B} \gamma$ lines together with measurements of the strengths of several other $\mathrm{H}_{2}$ lines. The data are discussed in terms of a simple shock-wave excitation model, and some estimates for the mass of the NGC 7027 molecular cloud are made.

\section{OBSERVATIONS}

The Ebert-Fastie grating spectrometer system described by Beckwith, Persson, Neugbauer, and Becklin (1978, Paper I) was used to make all the observations discussed in this paper. The system consists of an ambient temperature spectrometer together with a cold (77-K) continuously variable interference filter and an InSb detector at $55 \mathrm{~K}$. The spectral resolving power used in these observations was $\lambda / \Delta \lambda=1250(\Delta \lambda \sim 0.0017$ $\mu \mathrm{m})$ at $2.2 \mu \mathrm{m}$.

\section{a) Spatial Distribution}

A 5"-diam diaphragm was used on the Hale 5-m telescope on Palomar Mountain to measure the intensity

\footnotetext{
a) Present address: Astronomy Department, Cornell University, Ithaca, New York 14853.

b) Present address: Institute for Astronomy, University of Hawaii, Honolulu, Hawaii 96822.
}

of the $v=1 \rightarrow 0 S(1)$ transition $(\lambda=2.122 \mu \mathrm{m})$ at positions which lie along north-south and east-west lines passing through the center of the nebula. The nebular center was first located by maximizing the flux in a wideband detector system $(\Delta \lambda=0.4 \mu \mathrm{m}$ at $2.2 \mu \mathrm{m})$ with a 5 " diaphragm aligned with the spectrometer. The spectrometer was set to the $\mathrm{H}_{2}$ line wavelength by using a previously well established wavelength calibration; this calibration was checked several times by measuring the wavelength of the $\mathrm{HI} \mathrm{B} \gamma$ line $(n=7 \rightarrow 4)$ in the nebula. For most positions, only the fluxes at the line and at two continuum wavelengths separated by \pm 3 spectral resolution elements from the line center were measured. In no case was there a significant difference between the two continuum intensities. At the center of the nebula the peak line-to-continuum ratio was approximately unity.

The measurement positions were separated by onehalf of a spatial resolution element (2.5) and extended to positions off the nebula where the flux is negligible. Sky subtraction was accomplished by switching to a reference position $30^{\prime \prime}$ north or south of the measured position; this introduces no corrections for flux in the sky beam for any of the nebular positions. The visual seeing disk was always less than $2^{\prime \prime}$ in diameter, and the guiding uncertainties are estimated to have been $\sim 0^{\prime \prime} .5$. At least two independent measurements at each position on the nebula were made.

Alternately with the $\mathrm{H}_{2}$ measurements, the intensity of the $\mathrm{B} \gamma(2.16-\mu \mathrm{m})$ line was measured at each point, so that the $\mathrm{B} \gamma$ and $S$ (1) line strengths could be directly compared at the same spatial locations. The $\mathrm{B} \gamma$ line is strong and close to the wavelength of the $\mathrm{H}_{2}$ line. Seeing variations, guiding uncertainties, and variations in the diaphragm response profile affect the measurement of $\mathrm{B} \gamma$ in the same way as the measurement of the $\mathrm{H}_{2}$ line; 
TABLE I.. $\mathrm{H}_{2}$ emission from NGC 7027.

\begin{tabular}{lcccc}
\hline & & & $v \pm 2 \rightarrow 1$ \\
\hline $\mathrm{H}_{2}$ transition & $S(0)$ & $S(1)$ & $S(2)$ & $S(1)$ \\
$\operatorname{Flux}^{\mathrm{a}}\left(10^{-13}\right.$ & $4.6 \pm 0.7$ & $14 \pm 1$ & $<6(3 \sigma)$ & $1.0 \pm 0.5$ \\
$\left.\operatorname{erg~s}^{-1} \mathrm{~cm}^{-2}\right)$ & & & & \\
\hline \hline
\end{tabular}

a Fluxes in a $10^{\prime \prime}$-diam diaphragm centered on the $2-\mu \mathrm{m}$ peak. The quoted uncertainties are based on the fits of the instrumental profile to the spectra as described in the text. They do not include uncertainties due to seeing or calibration, both estimated to be $\sim 5 \%$ for all but the $S(2)$ line. For this line, atmospheric water vapor absorption strongly interferes with the measurement.

these uncertainties are therefore minimized in a direct comparison of the two spatial distributions.

\section{b) Line Strengths}

Intensity observations of the $v=1 \rightarrow 0 S(0), S(1)$, and $S(2)$, and $v=2 \rightarrow 1 S(1)$ lines were made at the center of the nebula using a $10^{\prime \prime}$-diam circular diaphragm on the 2.5-m telescope at Mount Wilson. The measurements were made in the same manner as those described in Paper I. For the three $v=1 \rightarrow 0$ lines, the intensity was measured at seven wavelengths around the center of the line. Four of these were at wavelengths \pm 2.2 and \pm 1.4 spectral resolution elements from the line center and defined the continuum baseline. Two measurements at \pm 0.5 resolution elements from the line center served to check the wavelength setting of the spectrometer, and one measurement at line center gave the line plus continuum intensity. Table I gives the resulting line strengths, where each entry was found by fitting these seven data points to the sum of a flat continuum plus an intrinsically narrow line convolved with the instrumental profile. For the $v=2 \rightarrow 1 S(1)$ line, only the line center and two continuum points were measured. No attempt was made to correct for atmospheric extinction. All the measurements were made at an air mass less than 1.5, and, as noted in Paper I, the atmosphere has little effect on the intensities of the $v=$ $1 \rightarrow 0 S(0)$ and $S(1)$ lines. There can be substantial uncertainty in measurements of the $v=1 \rightarrow 0 S(2)$ line $(\lambda=2.033 \mu \mathrm{m})$ because of extremely strong atmospheric water vapor absorption, and in fact the line was not detected. The equivalent width of the $v=2 \rightarrow 1 S(1)$ line is small, and only an upper limit to the line intensity is secure.

\section{RESULTS}

\section{a) Spatial Distribution of $\mathrm{H}_{2}$ Emission}

Figure 1 shows the results of the spatial distribution measurements of the $\mathrm{HI} \mathrm{B \gamma}$ and $\mathrm{H}_{2} v=1 \rightarrow 0 S(1)$ lines. The $\mathrm{B} \gamma$ intensity integrated over the nebula is in good agreement with the results of Merrill, Soifer, and Russell (1975). Also shown is the 6-cm map of NGC 7027 made with $2^{\prime \prime}$ resolution by Scott (1973). The solid curve in the top and bottom panels is a convolution of the radio map with a 5 " circular diaphragm moved along an east-west line. The intensity of the convolved profile was scaled to fit the central B $\gamma$ intensity. Similarly, the leftand right-hand panels contain the convolution of the radio data along a north-south line, compared to the $\mathrm{B} \gamma$ data.

It can be seen that in both directions the measured relative $\mathrm{B} \gamma$ intensities are in excellent agreement with the spatial distribution of the ionized gas as determined from radio continuum measurements. In both the north-south and east-west scans, the measured extent of the $\mathrm{H}_{2}$ line emission is greater than that of the atomic hydrogen line emission. This result is particularly evident between $5 "$ and 8 " away from the center of the nebula, where the $\mathrm{B} \gamma$ intensity has dropped to a small fraction of its central value, while the $\mathrm{H}_{2}$ intensity has dropped only slightly. This is the principal observational result of the spatial distribution measurements.

\section{b) $\mathrm{H}_{2}$ Line Strengths}

The results of the line strength measurements are given in Table I. The measurements of the $v=2 \rightarrow 1$ $S(1)$ transition resulted in a $3 \sigma$ upper limit to the line flux of $2.5 \times 10^{-13} \mathrm{erg} \mathrm{s}^{-1} \mathrm{~cm}^{-2}$. This limit, when combined with the $v=1 \rightarrow 0 S(1)$ line flux of $14 \times 10^{-13}$ erg s${ }^{-1} \mathrm{~cm}^{-2}$, yields an upper limit on the vibrational excitation temperature of $T_{\text {vib }} \lesssim 3200 \mathrm{~K}$ if the $\mathrm{H}_{2}$ molecules are collisionally excited; see Paper I for the numerical factors involved in this computation. This limit is consistent with the temperature of $2000 \mathrm{~K}$ found for the $\mathrm{H}_{2}$ lines in the Orion source (Paper I; Beckwith, Persson, and Neugebauer 1979; Simon et al. 1979).

\section{DISCUSSION}

\section{a) Spatial Distribution}

The major result of this work is that the observed $\mathrm{H}_{2}$ emission region in NGC 7027 is larger than the region of ionized gas. Some molecules could reside inside neutral clumps within the ionized region, as in NGC 6720 (Beckwith, Persson, and Gatley 1978), but in no case can these clumps be distributed in the same way as the ionized gas. We also note that the $\mathrm{H}_{2}$ emission is centered on the ionized region to within a few arcseconds.

The inferred physical sizes of the ionized and molecular regions, as deduced from the present data, depend on the detailed distribution of the $\mathrm{H}_{2}$ gas as a function of distance from the center of the nebula and on the diaphragm size used in the observations. To obtain a characteristic size for the $\mathrm{H}_{2}$ emission region, we calculated the expected intensity distributions for two simple models - a thin spherical shell and a uniformly filled sphere of optically thin emitting gas, each convolved with a $5^{\prime \prime}$ circular beam which represents the observing diaphragm. Comparison of the model results with the $\mathrm{H}_{2}$ data shows that a uniform sphere model cannot reproduce the east-west scan. However, the 


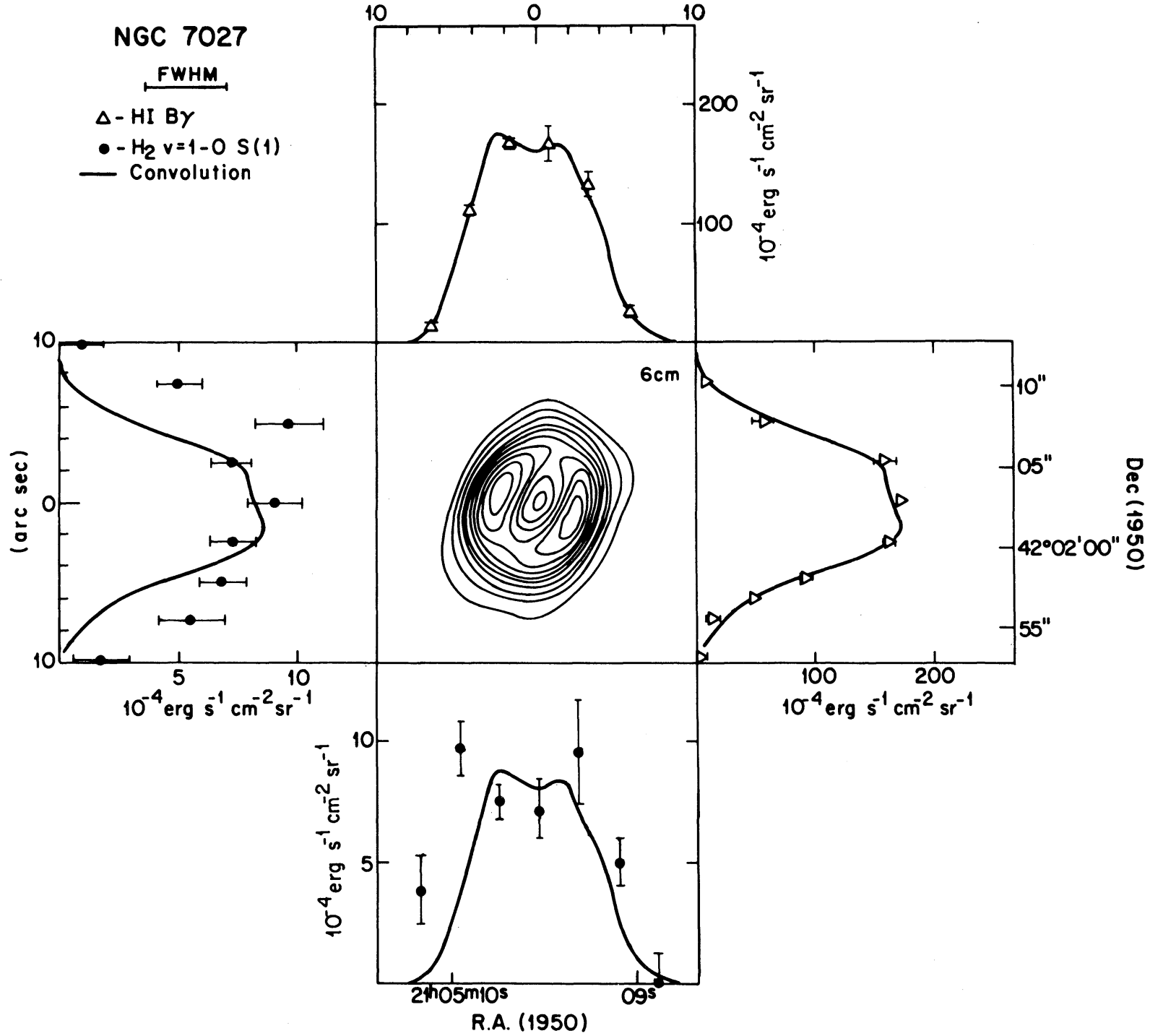

FIG. 1. Spatial distribution data for the $\mathrm{H}_{1} \mathrm{~B} \gamma$ and $\mathrm{H}_{2} S(1)$ lines together with the radio map of Scott (1973). The open symbols on the top and right-hand panels show the individual $\mathrm{B} \gamma$ data points along east-west and north-south scans. The solid curves in all four panels are the convolutions of the radio map with the 5"-diam diaphragm moved along east-west and north-south lines through the center of the nebula. The solid symbols on the bottom and left-hand panels show the corresponding $\mathrm{H}_{2}$ data; the $\mathrm{H}_{2}$ intensity scale has been adjusted to facilitate visual comparison of the extent of the $\mathrm{B} \gamma$ and $\mathrm{H}_{2}$ emission. The spatial resolution of 5" is indicated at upper left. All error bars are $\pm 1 \sigma_{m}$.

limb-brightened appearance of the data can be represented by a thin shell model; the best fit is shown in Fig. 2. The thickness of the shell was taken to be much smaller than $5^{\prime \prime}$, the exact value being immaterial. The north-south data cannot be fit well with either a shell or sphere model; the best fit shell is also shown in Fig. 2.

Although the east-west fit is reasonably good, in no case can it be said that a spherical shell uniquely represents all the $\mathrm{H}_{2}$ data. Rather, the actual $\mathrm{H}_{2}$ distribution is undoubtedly more complex. What the simple numerical experiment does show, however, is that the extended appearance of the $\mathrm{H}_{2}$ relative to the ionized gas emission may be due largely to limb brightening in a shell as seen with a relatively large observing diaphragm. We conclude that the excited $\mathrm{H}_{2}$ molecules need not reside in a region much larger than that of the ionized gas, whose diameter is $\sim 12^{\prime \prime}$.

\section{b) Excitation Mechanisms}

Pure radiative excitation of $\mathrm{H}_{2}$ molecules by ultraviolet fluorescence has been discussed by Black and Dalgarno (1976), Shull (1978), and Black (1978). Their calculations of the level populations which result from ultraviolet fluorescence predict a line intensity ratio $[v$ $=2 \rightarrow 1 S(1)] /[v=1 \rightarrow 0 S(1)]$ of $1 / 2$. The observed 

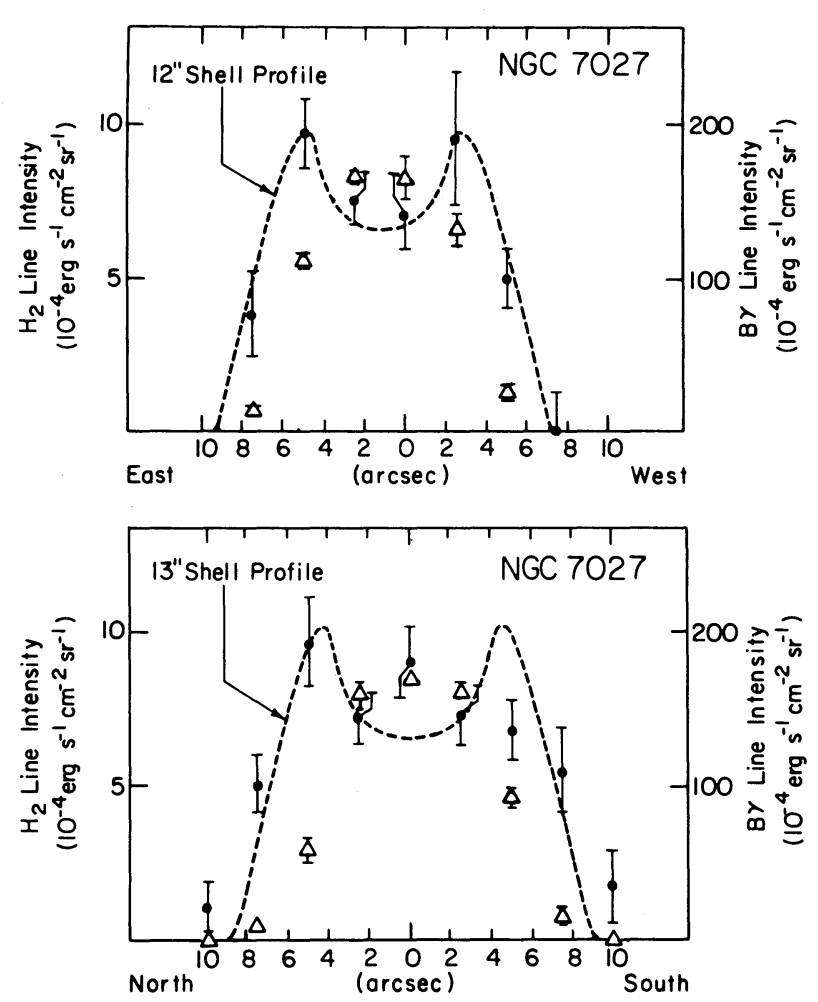

Fig. 2. The $\mathrm{H}_{2}$ data from Fig. 1 are repeated together with the bestfitting spherical shell models (dashed curves), which are the convolutions of a 5"-diam circular diaphragm with the projection of an optically thin shell of negligible radial thickness. See text for discussion.

$3 \sigma_{m}$ upper limit to this ratio is $1 / 4$ (Table I); if the $v=2$ $\rightarrow 1 S(1)$ line were one-half as strong as the $v=1 \rightarrow 0$ $S(1)$ line, it would have been detected with a signalto-noise ratio of 10 . Ultraviolet fluorescence alone is therefore inconsistent with the observed line ratio.

The level populations may be determined by collisional excitation, as seems to be the case in the Orion $\mathrm{H}_{2}$ source (Paper I). The present data are not sufficient to provide a test of this hypothesis, or a collisional temperature estimate, since only two lines, from different $\mathrm{H}_{2}$ species, were detected. It is, however, relatively easy to reproduce the observed line intensities by pure collisional excitation in a shock-heated region outside the ionized volume.

Shock-heated $\mathrm{H}_{2}$ can arise naturally in NGC 7027 from the expansion of the ionized nebula into the surrounding neutral cloud. The expansion velocity at the edge of the visible nebula is $22 \mathrm{~km} \mathrm{~s}^{-1}$ (Wilson 1950), and we assume the surrounding material is expanding at $\sim 9 \mathrm{~km} \mathrm{~s}^{-1}$ as determined from the width and shape of the $\mathrm{CO}$ emission line profile (Mufson, Lyon, and Marionni 1975). The inferred shock velocity $v_{s}$ of 13 $\mathrm{km} \mathrm{s}^{-1}$ is within the range of $10 \mathrm{~km} \mathrm{~s}^{-1} \leq v_{s} \leq 25 \mathrm{~km} \mathrm{~s}^{-1}$ which can excite the $\mathrm{H}_{2}$ molecules without completely dissociating them (Kwan 1977). For this shock velocity and an observed intensity of $8 \times 10^{-4} \mathrm{erg} \mathrm{cm}^{-2} \mathrm{~s}^{-1} \mathrm{sr}^{-1}$ in the $v=1 \rightarrow 0 S(1)$ line at the center of the nebula, we use Kwan's (1977) Fig. 2 to obtain a density estimate for the unshocked gas of $4 \times 10^{4} \mathrm{~cm}^{-3}$. We have assumed here that the front and back sides of the nebula contribute equally. This density is comparable to the mean electron density of $\sim 5 \times 10^{4} \mathrm{~cm}^{-3}$ within the nebula [see summary of Miller and Mathews (1972)]. This agreement lends support to the shock excitation picture.

The pressure necessary to maintain the shock can be provided easily by the expanding nebula. This pressure can be estimated from the rate of change of momentum of the material traversing the shock boundary: $P=$ $C m n v_{s}^{2}$, where $n$ is the unshocked volume density, $m$ is the molecular mass, and $C$ is a constant of order unity. Using $n$ and $v_{s}$ as given above. $P \sim 2 \times 10^{-7} \mathrm{dyn} \mathrm{cm}^{-2}$. The pressure associated with the kinetic energy density in the expanding ionized gas is of order $m_{p} n_{e} v_{e}^{2}=6 \times$ $10^{-7}$ dyn cm${ }^{-2}$, where $m_{p}$ is the proton mass, $n_{e}$ is the electron density, and $v_{e}$ is the nebular expansion velocity. These estimates show that the energy density needed to drive a strong shock wave into the surrounding cloud is available.

\section{c) Mass Estimates}

It is of interest to estimate the mass of neutral material surrounding NGC 7027. Mufson, Lyon, and Marionni (1975) derived a mass estimate of $\sim 1.4 M_{\odot}$ on the basis of their $\mathrm{CO}$ data and a standard $\mathrm{H}_{2} / \mathrm{CO}$ abundance ratio. As noted by these authors, a mass estimate of this kind may actually be impossible to make because of uncertainties in the ratio of ${ }^{12} \mathrm{CO}$ line strength to the column density of neutral material. Shields $(1978 \mathrm{a}, \mathrm{b})$ has shown that the carbon-to-hydrogen ratio in NGC 7027 is nearly ten times solar, and this will alter both the strength of the ${ }^{12} \mathrm{CO}$ line emission and the temperature and energy balance in the molecular cloud. In addition, the ${ }^{12} \mathrm{CO} /{ }^{13} \mathrm{CO}$ ratio is not known, and the degree of saturation of the ${ }^{12} \mathrm{CO}$ lines is thus uncertain. Finally, the fraction of carbon locked up in grains is unknown.

An alternative is to use the CO data only to give the size of the cloud. The mass follows from a volume integration over some density distribution, assumed spherically symmetric, from the inner radius of the molecular cloud, $R_{\min }$, to the outer radius $R_{\max }$.

Let the number density of molecules as a function of radius be $n(r)$. The mass is then

$$
M=4 \pi m_{2} \int_{R_{\min }}^{R_{\max }} r^{2} n(r) d r,
$$

where $m_{2}$ is the mass of a $\mathrm{H}_{2}$ molecule. One way of estimating $n(r)$ is to take $n\left(R_{\min }\right) \sim 4 \times 10^{4} \mathrm{~cm}^{-3}$ based on the shock model discussed in Sec. IV b and to assume a plausible functional form for the radial dependence. This clearly involves considerable uncertainty, and we prefer the following method. We estimate the amount of dust associated with the nebula from the fluctuations in reddening over the face of the object and relate this 
to the column density of hydrogen associated with the nebula. This idea was used by Mufson et al. as a check on their density estimate from the $\mathrm{CO}$ data. Miller and Mathews (1972) found fluctuations in the reddening across the nebula amounting to $\delta E(B-V) \sim 0.15 \mathrm{mag}$; this gives a lower limit to the amount of dust associated with the nebula. Recently, Atherton et al. (1979) have reported a more detailed study of the reddening variations across the face of the nebula. Their results show that the relative extinction at $\mathrm{H} \alpha$ varies by $\gtrsim 1.5 \mathrm{mag}$ from the center to edge; this corresponds to $\delta E(B-V)$ $\sim 0.6$ mag. Taking the interstellar ratio of $\mathrm{H} \mathrm{I}$ column density to color excess as $N / E(B-V)=7 \times 10^{21}$ $\mathrm{cm}^{-2} \mathrm{mag}^{-1}$ (Jenkins and Savage 1974), we obtain $N$ $\sim 4 \times 10^{21} \mathrm{~cm}^{-2}$. The (neutral) column density $N$ is related to the (molecular) density distribution $n(r)$ by

$$
N=2 \int_{R_{\min }}^{R_{\max }} n(r) d r .
$$

Dividing Eq. (1) by Eq. (2) then removes some of the uncertainty due to $n(r)$ in the mass estimate:

$$
M=4 \pi m_{2} N \int_{R_{\min }}^{R_{\max }} r^{2} n(r) d r / \int_{R_{\min }}^{R_{\max }} n(r) d r,
$$

or

$$
M / M_{\odot}=(D / 1.8)^{2} \int_{1}^{5} n(r) r^{2} d r / \int_{1}^{5} n(r) d r
$$

where $D$ is the distance to NGC 7027 in kpc. The limits of integration are expressed in units of 6 " and are taken from our $\mathrm{H}_{2}$ data and the $\mathrm{CO}$ data of Mufson et al. A reasonable guess for the functional form of $n(r)$ is $n(r)$ $\propto r^{-p}$, in which case the ratio of the two integrals is 5 for $p=2$ and 2.4 for $p=4$. The slowly varying nature of the ratio means that the dominant uncertainty may be not the exact form of $n(r)$ but rather the assumption that the amount of gas has the same proportion to extinction as the interstellar value. If the distance to NGC 7027 is 1.8 kpc, we estimate that $M / M_{\odot} \sim 3$, with an uncertainty of the order of a factor of 3 .

With a mass estimate of $3 M_{\odot}$ and a density of $n(r)=$ $4 \times 10^{4}\left(r / R_{\min }\right)^{-p} \mathrm{~cm}^{-3}$, Eq. (1) is satisfied for $p=3.9$. Atherton et al. (1979) have studied the distribution of light in the faint reflection nebula surrounding NGC 7027 and have found that the grain number density falls off approximately as $r^{-3}$. The agreement between our results and those of Atherton et al. for the density structure in the molecular envelope surrounding NGC 7027 is encouraging. Future mapping of the NGC 7027 molecular cloud in higher-frequency $\mathrm{CO}$ lines should be capable of giving another estimate of $p$. It should then be possible to tie together the density and extinction in the cloud with its mass.

\section{SUMMARY}

(1) Spatial mapping of the $v=1 \rightarrow 0 S(1)$ line of $\mathrm{H}_{2}$ and the $\mathrm{B} \gamma$ line of $\mathrm{HI}$ in NGC 7027 shows that the excited $\mathrm{H}_{2}$ molecules reside near the edge or just outside the ionized nebula.

(2) Measurements of the $v=2 \rightarrow 1 S(1)$ and $v=1 \rightarrow$ $0 S(1)$ line intensities at the center of the nebula rule out pure ultraviolet fluorescences as the dominant excitation mechanism for the $\mathrm{H}_{2}$ molecules.

(3) The $\mathrm{H}_{2}$ emission could result from shock-heated gas just outside the ionized nebula. In this case the density in the unshocked cloud is $\sim 4 \times 10^{4} \mathrm{~cm}^{-3}$.

(4) A rough estimate of the mass of the NGC 7027 molecular cloud gives $M \sim 3 M_{\odot}$, with an uncertainty of a factor of 3 .

We thank our night assistant Juan Carrasco for help with the observations, and we are grateful to Dr. T. N. Gautier, III, the referee, for his helpful suggestions and for pointing out an error in the original text. This work was supported in part by NSF Grant AST 77-20516 and NASA Grant NGL 05-002-207.

\section{REFERENCES}

Atherton, P. D., Hicks, T. R., Reay, N. K., Robinson, G. J., Worswick, S. P., and Phillips, J. P. (1979). Astrophys. J. 232, 786.

Beckwith, S., Persson, S. E., and Gatley, G. (1978). Astrophys. J. Lett. 219, L33.

Beckwith, S., Persson, S. E., and Neugebauer, G. (1979). Astrophys J. 227, 436

Beckwith, S., Persson, S. E., Neugebauer, G., and Becklin, E. E. (1978). Astrophys. J. 223, 464 (Paper I).

Black, J. H. (1978). Astrophys. J. 222, 125.

Black, J. H., and Dalgarno, A. (1976). Astrophys. J. 203, 132.

Jenkins, E. B., and Savage, B. D. (1974). Astrophys. J. 192, 447.

Kwan, J. (1977). Astrophys. J. 216, 713.

Miller, J. S., and Mathews, W. G. (1972). Astrophys. J. 172, 593.
Merrill, K. M., Soifer, B. J., and Russell, R. W. (1975). Astrophys. J. Lett. 200, L37.

Mufson, S. L., Lyon, J., and Marionni, P. A. (1975). Astrophys. J. Lett. 201, L85.

Scott, P. F. (1973). Mon. Not. R. Astron. Soc. 161, 35P.

Shields, G. A. (1978a). Astrophys. J. 219, 559.

Shields, G. A. (1978b). Astrophys. J. 219, 565.

Simon, M., Righini-Cohen, G., Joyce, R. R., and Simon, T. (1979). Astrophys. J. Lett. 230, L175.

Shull, J. M. (1978). Astrophys. J. 219, 877.

Treffers, R. R., Fink, V., Larson, H. P., and Gautier, T. N., III. (1976). Astrophys. J. 209, 793.

Wilson, O. C. (1950). Astrophys. J. 111, 279. 